\title{
Editorial
}

\section{Thoughts on life with untested and adequately tested chemicals}

Medical expert witnesses in courts of law take adversarial positions and present data to support either the defence or the prosecution, or one of the litigants in a compensation claim. By nature their views are biased. Apart from being a witness in court, you may be asked to counsel a solicitor about the correctness of a compensation claim for alleged toxic damages. Here there is no need to be biased; a well researched yes or no may guide the case in the right direction and help to save expenditure of personnel and money. Counselling in a concrete case may also help to discover that information, even on a well researched toxic substance, is peppered with black holes. The lack of precedent, experimental, clinical, or epidemiological, does not exclude the possibility that the claim is justified but it makes its justification impossible. The claimant's case may be the first or through the absence of a precedent many previous cases remain unrecognised and unreported. Thus the system is as heavily biased against the claimant as the acceptance of a claim on the basis of a possible (as opposed to impossible) association beween injury and exposure would be biased against the employer. Whereas in the case of an untested chemical the chance of an unjust decision is as great as the number of possible toxic actions, that is decreased by toxicity tests, research on biochemical interaction, and clinical and epidemiological surveillance.

\section{Life with untested chemicals}

According to the United States National Academy of Sciences the available data base permits complete health hazard assessment for $6.8 \%$ and partial health hazard assessment for $16.4 \%$ of 17202 chemicals present in pesticides, cosmetics, drugs, and food additives. ${ }^{\prime}$ For a surprisingly large number of chemicals $(44 \%)$ there is no toxicity information at all. Commercial products that do not fall into these four categories have even worse testing records. The proportion of chemicals with data permitting complete health hazard assessment is zero and permitting partial assessment, $11 \%$. No toxicity information is available on $80 \%$ of the chemicals. The proportion of untested or inadequately tested chemicals may be similarly high in the groups of intermediates and byproducts that do not reach consumer goods but are potential occupational hazards.

The reasons why only a small proportion of industrially useful chemicals has been adequately tested are expense and insufficient testing facilities. Testing is not able to keep step with new developments and even less able to decrease the backlog. The economical use of testing facilities means that tests are almost exclusively carried out on new chemicals and only when required by law. A chemical gets a low priority grading when the volume of production or release rate into the work or general environment is low. Moreover, toxicity testing is not always comprehensive. Owing to the availability of in vitro and short term mutagenicity (and related) tests, substantially more chemicals are tested for carcinogenic potential than for systemic toxicity.

Though the sheer number of chemicals produced and used without adequate risk assessment is alarming, there is nothing to suggest that the alarm is justified. Perhaps the increasing use of structure activity relations for the identification of potential toxic hazards successfuly eliminated all those compounds from the industrial field which could have been the cause of noticeable disasters. Another explanation is that the list of untested or inadequately tested compounds includes some toxic ones, but routine industrial practice and consumer use are not associated with toxic exposure. Alternatively, the list may contain some toxic compounds that cause illness but the connection has remained unrecognised because toxic reactions can be explained with the effects of known chemical, physical, or microbiological agents.

Historical evidence indicates that when a large number of people become ill in the same district and at the same time, a microbiological origin is usually the first and the most attractive hypothesis. This happened when, in 1930, about 50000 people developed different degrees of leg paralysis in the southern and midwestern states of the United States. The disease was diagnosed as neurosyphilis, polyomyelitis, or toxic influenza, and the role of tri-ortho-cresyl phosphate which was a contaminant in Jamaica ginger 
extract was discovered only later. ${ }^{2}$ The sporadic occurrence of a "contagious weird disease," which later became known as Minamata disease, was concealed and patients were secretly admitted to municipal hospital isolation wards. In 1956, three years after the first occurrence, the concurrent admission of four patients prompted a local hospital to report the outbreak of an unusual disease of the nervous system to the Minamata Health Center. Subsequent investigation found about 30 other people with a similar disorder. All were placed in a ward for infectious diseases. Six months later a study group suggested that a toxic metal, possibly selenium, manganese, or thallium, was the causative agent. ${ }^{34}$ By a stroke of luck in 1958, Dr D McAlpine, who almost two decades before had seen methylmercury intoxicated patients in London, visited the Minamata patients. In the absence of adequate chemical analytical data he was unable to make a positive diagnosis but he raised the possibility of methylmercury intoxication. ${ }^{5}$ The redirected search for the causative agent was so successful that in 1959 the Study Group on Minamata Disease was able to announce that mercury released with wastes from the nearby acetaldehyde plant and accumulated in seafood was the causative agent, and methylmercury was identified in seafood and in the patient's blood and hair. ${ }^{34}$

The pattern of occurrence in the tri-ortho-cresyl phosphate epidemics (drinking habits or residence near to certain drug stores known to violate prohibition laws) or in the Minamata epidemics (fishermen and close relatives) helped more than anything else to identify the vehicle containing the toxic agent. Sporadic occurrence and the lack of diagnostic case to case comparison should have made identification of the toxicologically unknown tri-ortho-cresyl phosphate more difficult or impossible. Though the clinical characteristics of methylmercury intoxication in occupationally exposed victims had been described by Hunter et al a long time before the outbreak in Minamata, the clinical findings were not widely known. ${ }^{6}$ Moreover only research prompted by the Minamata experience led to the appreciation of the bioaccumulative potential of methylmercury.

The tri-ortho-cresyl phosphate and Minamata epidemics showed how difficult it is to make deductions about exposure from clinical findings. Even with the knowledge of the chemicals present in a plant, the medical officer will scarcely attempt to explain a clinical condition with exposure to a chemical with no known toxic effects. It is more likely that non-chemical aetiological factors, or a chemical known to produce the illness, will be blamed. Nevertheless, now and again the responsibility of an untested chemical for an illness may be raised. As the chemical is untested, a causal relation can be claimed only by intuition and be denied by equating the lack of data with the lack of effects. Therefore a reasonable compensation claim cannot be justified or an unreasonable one declared impossible. Actually, the more we know about the toxicity of a chemical, the easier we may refute an unjustified claim. The following twin examples, one imaginary and the other taken from life, will illustrate this point.

Two neonates died shortly after birth from congenital absence of kidneys. One of the mothers was exposed to an untested chemical and the other to a mercury salt. When the responsibility of the untested chemical is claimed, the disclaimer can argue vehemently against such a teratogenic action but he must admit that (i) in the absence of any toxicity investigation the possibility cannot be excluded that the chemical is teratogenic and (ii) the dose response relation might be such that a teratogenic effect is possible at the exposure level of the mother.

In the other case the well known necrotic effect of mercurial salts is used as an argument to support the connection between exposure and the congenital defect. ${ }^{7}$ The disclaimer now has a ball to kick. He can point out that (i) teratological changes, such as the absence of kidneys, indicates generative changes in undifferentiated cells whereas mercurial kidney damage is a degenerative change in highly differentiated proximal tubular cells; (ii) animal experiments indicated that at birth the kidneys are insensitive to the nephrotoxicity of $\mathrm{HgCl}_{2}$ and the full nephrotoxic effect become evident when postnatal structural maturity is attained $^{89}$; (iii) mercurial fetotoxicity, such as resorption, retarded fetal growth, and fetal oedema, is conditional on the presence of indicators of severe maternal toxicity, such as weight loss, kidney damage, diarrhoea, tremor, and somnolence ${ }^{10}$; (iv) in severely intoxicated pregnant hamsters the frequency of malformations does not exceed $5 \%$ of the surviving fetuses and the urogenital system is never affected ${ }^{11}$; (v) additional experiments indicated that the placenta forms a barrier against the passage of mercuric mercury from the maternal blood to the fetus ${ }^{12}$ and therefore the effects of maternal exposure to an inorganic mercury salt on the fetus results from severe maternal intoxication ${ }^{13}$; (vi) this view was supported by a clinical observation of a woman who ingested 2.5 $\mathrm{g}$ of mercuric chloride to induce abortion. She soon developed complete anuria, but aborted an anatomically normal fetus 13 days after ingestion. ${ }^{14}$ Thus all the available evidence, including the absence of maternal renotoxicity, contradicts the role of an inorganic mercury salt in impaired fetal development.

Life with well known and adequately tested chemicals

Nevertheless, adequate testing and animal experimen- 
tation, even when supplemented with epidemiological studies, does not guarantee an automatic answer to every problem. To dispel such an overoptimistic view one must consider only the controversy concerning the effects of lead on early mental and behavioural development. Less politically motivated subjects, such as the effects of elemental mercury on female reproduction and the aetiological role of lindane (the gamma isomer of hexachlorobenzene) in aplastic anaemia, may be more illustrative.

The effect of exposure to mercury vapour on female reproductive function is a grey area. There are few publications and those have questionable reliability. One report implies that in the dental profession exposure to mercury vapour increased the frequency of reproductive failure and that there is a positive correlation between mercury in hair and reproductive failure or menstrual disorders. ${ }^{15}$ Unfortunately the authors did not follow sound epidemiological practice or analyse the data critically. Exposed and control subjects were not matched according to age and medical history, including the number of pregnancies; time lapses between pregnancy and the start of exposure or the time of mercury analysis were not given; and reproductive failures were subdivided to abortion, stillbirths, and malformations for the exposed but not for the control group. As the proportion of women who had reproductive failure does not differ significantly in the two groups, a correlation between mercury concentration in hair and reproductive failure seems doubtful. Furthermore, mercury in hair correlated both with exposure years and the number of amalgams prepared a week, which implies that the number of weekly prepared amalgams had also increased with years spent in the profession.

There are some other reports on the effects of exposure to mercury vapour on the incidence of menstrual disorders or abortion. Laraview (quoted by EPA $^{16}$ ), Marinova et al (quoted by Schrag and Dixon $^{17}$ ), Panova and Dimitrov (quoted by De Rosis $e t$ $\left.a l^{18}\right)$ and De Rosis et al questioned women about their periods. Whereas the first three studies from the USSR found an increased incidence of menstrual disorders in women exposed to mercury vapour, the study of De Rosis et al from Italy gave an equivocal answer. These authors did not give the actual frequencies of premenstrual syndrome for exposed and non-exposed women and there was no significant difference in the frequencies of abnormal menstrual cycles (polymenorrhoea or oligomenorrhoea), the "overall pathology of pregnancy (spontaneous abortion, premature delivery, threat of abortion, or premature delivery), birth weight, or malformations." Significant differences were found only when the authors compared the proportion of women who noticed an exacerbation of premenstrual syndrome or change in menstrual cycle during employment. The impact of this finding, however, is greatly weakened by the numbers which show that, although $43 \%$ of the exposed women noticed a change in the menstrual cycle only $35 \%$ had abnormal cycles. The most feasible exlanation is that exposure to mercury in some women was associated with the normalisation of the menstrual cycle.

Difference in the degree of exposure may explain the difference between the findings of De Rosis et al and the three USSR studies. The exposure of the cohort of De Rosis did not exceed $0.01 \mathrm{mg} / \mathrm{m}^{318}$ whereas Laraview's cohort frequently had symptoms of mercury vapour intoxication. ${ }^{16}$ By contrast with De Rosis et al, Laraview or Wiksztrajtis (both quoted by EPA $^{6}$ ) reported an increased incidence of abortion and Mishanova et al claimed that in women exposed to "microdoses" (probably less than $1.0 \mathrm{mg} / \mathrm{m}^{3}$ ) of mercury vapour the frequencies of eclampsia, protracted labour, and placental haemorrhage increased in a dose dependent way. ${ }^{19}$ This report, however, is defective on various grounds: (i) only the differences between exposed and non-exposed groups are shown and not the dose response relation; (ii) controls were not matched or data stratified on possible confounding factors; and (iii) the selection of controls may be questioned on the basis of significant increase in the frequency of anaemia, a non-mercurial effect, in the exposed group.

Despite the shortcomings of these studies, historical evidence supports the view that toxic exposure to mercury vapour at a relatively early gestation age has an abortifacient effect. The question is the dose response relation. The abortifacient effect is indicated by the case of a woman chronically intoxicated by mercury vapour whose pregnancies ended unfavourably. After she recovered from overt mercury poisoning, she gave birth to a healthy child (see EPA ${ }^{6}$ ). That mercury vapour intoxication has no such effect during late pregnancy is shown by another case in which an acutely intoxicated woman delivered a healthy baby 26 days later. ${ }^{20}$

Though one rightly criticises the standard of published epidemiological studies, it would be unjustified to reject the possibility that without the well known signs of erethism, mercury vapour can adversely affect the menstrual cycle or fetal development. Rejection is unjustified on two counts. Firstly, experiments on rats exposed to a high mercury vapour concentration support-at least partly-these assumptions. It was reported from Poland that repeated daily exposure of female rats to $2.5 \mathrm{mg} \mathrm{Hg} / \mathrm{m}^{3}$ increased the duration of the oestrus cycle and decreased the number of live fetuses and birth weight but caused no malformations. ${ }^{21}$ Secondly, neither the review of Koos and Longo ${ }^{14}$ nor monographs such as the Handbook on the Toxicology of Metals ${ }^{22}$ or 
Reproductive and Developmental Toxicity of Metals, ${ }^{23}$ mention any publication from Western Europe or North America that contradicts or confirms these assumptions. For some reason and despite the number of women of child bearing age exposed to mercury vapour such studies are lacking and the reproductive effects of mercury vapour at prevailing exposure levels remain a possibility.

The lindane controversy is of a completely different nature. Loge, who presented two cases of aplastic anaemia after exposure to lindane, pointed out that in the absence of specific in vitro or in vivo tests which can link a suspected exposure with a particular example of aplastic anaemia the physician is left with the historical approach to determine aetiology. ${ }^{24}$ Thus onset followed exposure in both cases described by Loge. A mother recalled that her 8 year old daughter, who subsequently died as a result of aplastic anaemia, used to spend two hours in another child's home where a lindane burner was kept in use for 24 hours a day in the play room. The fate of the friend was not mentioned. The second victim was a 52 year old man who in early September 1962 bought a lindane vapouriser and in mid-October noticed the appearance of scattered echymoses. West described four other cases, three with fatal outcome; the fourth was a 4 year old child whose illness was diagnosed as atypical leukaemia. ${ }^{25}$ According to Hans, by 1976 there were at least 11 published cases with a definite temporal relation between exposure to lindane and the onset of aplastic anaemia. ${ }^{26}$ There were at least ten others in which exposure to lindane was accompanied by exposure to additional materials suspected of causing aplastic anaemia.

Despite these case reports the latest edition of Patty's Industrial Hygiene and Toxicology $y^{27}$ does not accept the view that lindane can produce haematological disorders through the toxic suppression of haematopoiesis. The reason for this is that case reports can only suggest the possibility of a hazard. Even repeated experience of a temporal relation, though it makes the possibility more tenable, does not prove an association between exposure and disease. Such an association can be confirmed only by epidemiological studies. Two epidemiological studies approached the problem from different directions. A cross sectional study compared occupationally exposed and unexposed populations. ${ }^{28}$ They were matched by sex, race, and year of age. The exposed population showed no evidence of pancytopaenia or reticulopaenia, the hallmark of aplastic anaemia. A case-control study of the association between fatal aplastic anaemia and occupations entailing exposure to pesticides found that the use of chlorinated hydrocarbon pesticides (including lindane) did not increase the mortality from aplastic anaemia. ${ }^{29}$ Nevertheless, severe lindane intoxication may cause a temporary anaemia. After they sprinkled the ground and walls of their houses, their bedcovers, clothes, and sometimes their bodies with a mixture of hexachlorobenzene isomers, 79 people developed disorders of the gastrointestinal, cardiac, and nervous systems. Five patients were admitted to hospital and one died four days after admission. The other four had diminished red cell counts, a proportionate decrease in haemoglobin concentration, lack of reticulocytes, and normal platelet counts. After the poor and inadequately fed victims had been given a high protein diet and other treatments, they improved rapidly. ${ }^{30}$

Conflicting historical and epidemiological evidence is not rare. The suspension of the product licence of benoxaprofen (Opren) was based on historical evidence which indicated that treatment with this drug had been responsible for severe, frequently fatal cholestatic jaundice. ${ }^{31}$ The case reports were contradicted by five reports based on the treatment of 764 patients. $^{32-36}$ Moreover, Inman, who had identified, through prescription event monitoring, 24000 patients treated with benoxaprofen, found that $50 \%$ of the 54 cases of jaundice or renal failure was definitely unrelated to treatment and association was unlikely in 15 and possible in 11 cases. ${ }^{37}$ The evidence reached the level of probability only in one (non-fatal) case. Thus contrary to the historical evidence these reports raised serious doubts about the connection of the drug and deaths. Unfortunately, the two epidemiological studies on lindane did not give the same degree of assurance. Firstly, both studies were small and their target was occupationally exposed individuals, whereas the victims of the case reports were exposed to lindane as a result of fumigation in private dwellings. Thus the possibility cannot be ruled out that non-occupational exposure exceeded occupational exposure and non-occupationally exposed individuals were included in the control populations. Moreover, neither the cross sectiona ${ }^{28}$ nor the case-control study ${ }^{29}$ quantified (level and years of) exposure and the case-control study did not distinguish between lindane and other chlorinated hydrocarbons.

The lack of, or limitation of, exposure data, actual concentrations, and duration of exposure is a serious impediment of occupational and environmental epidemiology. Such information for an occupational study requires more than the acquiescence of the management. It requires active participation. It is not by accident that the two epidemiological studies on lindane and the only Western European study on the effect of mercury vapour on female reproduction came from university departments. Modern management is willing to obey laws and regulations which aim to prevent the recognised adverse effects of toxic com- 
pounds, but there is no incentive for the identification of so far unknown or unproved risks. A historical example for this attitude, hopefully less typical now than it was $\mathbf{4 0}$ years ago, is the experience of Harriet $L$ $\mathrm{Hardy}^{38}$ on beryllium disease. In May 1946 she was asked to read a paper describing the illness in a fluorescent lamp plant which had been backed up by complex insurance policies to handle catastrophes. It is worth quoting a few sentences from her reminiscences.

"Once news of this paper was public, a series of almost comic attempts were made by the industry and its insurer to prevent my reading it or at least to change the contents of my paper, hoping to turn attention away from the 'new' occupational disease. During my reading, a court stenographer typed away, seated just below the place where I stood. Company officials were at hand to question me making me feel excited and, ironically, amused, as these maneuvers failed to suppress the 1946 paper I read. ... An insurance physician, for reasons one might guess, ... insisted that the sick workers suffered from sarcoidosis . . . due to a virus washed into the plant from nearby ocean waves which swept across the marshes at high tide. One can only guess what economic pressures led this Harvard-trained MD to present such a notion."

For every story like this, there is probably another which shows the willingness to accept the bad news in order to avoid a disaster. Hostility or acceptance, however, does not change the absence of set procedures by which the unknown effect of occupational exposure to a chemical may be recognised. An improvement on the field of pesticides may be initiated by the draft report of the chairman of the House of Commons Agriculture Committee which called for a centralised system to coordinate all reports of pesticide poisoning. ${ }^{38}$ If such a system is extended to record all illnesses occurring during or within a certain time after exposure, not only will the epidemiology of known toxic effects be made easier, but so will the discovery of new ones. The aim is to have a system for the toxic effects of occupational hazards similar to that which has been operating for the recognition of adverse drug reactions. Doctors are asked to report suspected adverse drug reactions on reply-paid "yellow cards" and prescription-event monitoring is a way to study systematically the incidence of events that occur while patients are taking the drug or after they have stopped it. ${ }^{37}$

There is no similar system for occupational hazards. Within a large firm medical records may be related to exposure, but even in this case data are dispersed between different firms. Moreover, a change of employment or working for small firms without medical departments presents unsurmountable problems for establishing whether exposure to a certain chemical is associated with an increased incidence of a certain disease or deviation from normal physiological functions. It cannot be difficult to have a computerised central register of personal cards which will give the history of diseases (and pregnancy outcomes for women in the childbearing age) and of exposure. Having such a register in the main industrial countries would delete many question marks in our knowledge. Case reports would not be influenced by hesitancy to report or eagerness to publish and the population size of epidemiological studies could be expanded. It certainly would help to settle more satisfactorily compensation claims and to correct occupational health standards when and where necessary.

MRC Toxicology Unit,

L MAGOS

Carshalton, Surrey SM5 $4 E F$.

\section{References}

1 Nelson N. Perspectives on testing for toxic action. Environ Health Perspect 1987;75:97-103.

2 Turley LA, Shoemaker HA, Bowden DT. Jake paralysis. Norman, Oklahoma: University of Oklahoma Press, 1931.

3 Environmental Agency of Japan. Pollution related diseases and relief measures in Japan. Tokyo: Printing Bureau, Ministry of Finance, 1972.

4 Tsubaki T, Irukuyama K, eds. Minamata disease. Tokyo: Kidansha Ltd, 1977.

5 McAlpine D, Araki S. Minamata disease. An unusual neurological disorder caused by contaminated fish. Lancet 1958;ii:629-31.

6 Hunter D, Bomford RR, Russel DS. Poisoning by methyl mercury compounds. Q J Med. 1940;9:193-214.

7 Fogg E, Jennings A. Mercury peril from soap manufacture. New Scientist 1985; No 1456:9.

8 Wachstein M, Robinson M. Neonatal resistance to nephrotoxic renal tubular necrosis in the rat. Fed Proc 1965;24:619.

9 Daston GP, Kavlock RJ, Rogers EH, Carver B. Toxicity of mercuric chloride to the developing rat kidney. I. Postnatal ontogeny of renal sensitivity. Toxicol Appl Pharmacol 1981;71:24-41.

10 Gale TF, Ferm VH. Embryopathic effects of mercuric salts. Life Sciences 1971;10:1341-7.

11 Gale TF. Embryopathic effects of different routes of administration of mercuric acetate in the hamster. Environ Res 1974;8: 207-13.

12 Clarkson TW, Magos L, Greenwood MR. The transport of elemental mercury into fetal tissues. Biol Neonate 1972;21: 239-44.

13 Holt $\mathrm{D}$, Webb $M$. The toxicity and teratogenicity of mercuric mercury in the pregnant rat. Arch Toxicol 1986;58:243-8.

14 Koos BJ, Longo LD. Mercury toxicity in pregnant women, fetus, and new born infant. Am J Obstet Gynecol 1976;126:390-409.

15 Sikorski R, Juszkiewicz T, Paszkowski T, Szprengier-Juszkiewicz $T$. Women in dental surgeries: reproductive hazards in occupational exposure to metallic mercury. Int Arch Occup Environ Health 1987;59:551-7.

16 Environmental Protection Agency. Mercury health effects update. (Health issue assessment). Research Triangle Park: North Carolina, 1984.

17 Schrag SD, Dixon RL, Reproductive effects of chemical agents. In: Dixon RL, ed, Reproductive toxicology. New York: Raven Press: 1985:301-19. 
18 De Rosis F, Anastasio SP, Selvaggi L, Beltrame A, Moriani G. Female reproductive health in two lamp factories: effects of exposure to inorganic mercury vapour and stress factors. $\mathrm{Br} J$ Ind Med 1985;42:488-94.

19 Mishinova VN, Stepanva PA, Zarudin VV. Pregnancy and parturition in women inhaling microdoses of metallic mercury vapors in workplaces. Gig Tr Prof Zabol 1980;2:21-23. (In Russian.)

20 Lien DC, Todoruk DN, Rajani HR, Cook DC, Herbert FA. Accidental inhalation of mercury vapour: respiratory and toxicological consequences. Can Med Assoc J 1983;129:591-4.

21 Baranski B, Szymczyk I. Effects of mercury vapor upon reproductive functions of female white rats. Med $\operatorname{Pr} 1973 ; 24: 248-61$. (In Polish.)

22 Berlin M, Mercury. In: Friberg L, Nordberg GF, Vouk VB, eds. Handbook on the toxicology of metals. Vol 2. Amsterdam: Elsevier, 1986:387-445.

23 Clarkson TW, Nordberg GF, Sager PR, eds. Reproductive and developmental toxicity of metals. New York: Pelenum, 1983.

24 Loge JP. Aplastic anemia following exposure to benzene hexachloride. JAMA 1965;193:104-8.

25 West I. Lindane and hematologic reactions. Arch Environ Health 1967;15:97-101.

26 Hans RJ. Aplastic anemia associated with $\gamma$-benzene hexachloride. JAMA 1976;236:1008-9.

27 Deichmann WB. Hexachlorocyclohexane, In: Clayton GD,
Clayton FE, ed. Patty's industrial hygiene and toxicology. Vol 2B. 3rd ed. New York: John Wiley \& Sons, 1981:3740-9.

28 Milby TH, Samuels AJ. Human exposure to lindane. Comparison of an exposed and unexposed population. J Occup Med 1971;13:256-8.

29 Wang H, Grufferman S. Aplastic anemia and occupational pesticide exposure: a case control study. J Occup Med 1981;23:364-6.

30 Danapoulos E, Melissinos M, Katasas G. Serious poisoning by hexachlorocyclohexane. Arch Ind Hyg Occup Med 1953;8: 582-7.

31 Anonymous. Benoxaprofen. Br Med J 1982;285:459-60.

32 Allen BR, Littlewood SM. Side effects of benoxaprofen. $\mathrm{Br}$ Med J 1982;285:209.

33 Dunn EC. Side effects of benoxaprofen. Br Med J 1982;285:170.

34 Etherington JD. Side effects of benoxaprofen. Br Med J 1982;285:135.

35 Halsey JP. Benoxaprofen: side effects profile in 300 patients. $\mathrm{Br}$ Med J 1982;284:1365-8.

36 Hicklin JA. Side effects of benoxaprofen. Br Med J 1982;285:135.

37 Inman WHW. Risks in medical interventions. In: Cooper MG, ed. Risk. Man-made hazards to man. Oxford: Clarendon Press, 1985:35-53.

38 Hardy HL, Beryllium disease: a clinical perspective. Environ Res 1980;21:1-9.

39 Coggon D. Glasnost on pesticides. Be Med J 1988;296:81-2. 